\title{
The significance of preoperative serum carcinoembryonic antigen levels in the prediction of lymph node metastasis and prognosis in locally advanced gastric cancer: a retrospective analysis
}

Keshen Wang ${ }^{1+}$, Xiangyan Jiang ${ }^{2+}$, Yanxian Ren ${ }^{1}$, Zhijian $\mathrm{Ma}^{2}$, Xiaocheng Cheng ${ }^{1}$, Fan Li', Jingying Xiao ${ }^{1}$, Zeyuan $\mathrm{Yu}^{1,2}$ and Zuoyi Jiao ${ }^{1,2^{*}}$

\begin{abstract}
Background: In this study, we aimed to investigate the preoperative serum carcinoembryonic antigen (CEA) in the diagnosis of positive lymph node metastasis (LNM), and to evaluated the relationship between CEA and survival in patients with locally advanced gastric cancer (LAGC).

Methods: The significance of the preoperative serum CEA level for the diagnose of LAGC and prediction of LNM was determined using the receiver operating characteristic (ROC) curve. The areas under the ROC of CEA were compared with those of other tumor markers or imaging examination including $\mathrm{CT}$ and MRI. Logistic regression was utilized to identify the risk factors predicting positive LNM. Independent prognosis factors were evaluated using univariate and multivariate COX regression analyses.
\end{abstract}

Results: The ROC curves showed that the AUCS of CEA, CA199, and CA125 for diagnosing LAGC were 0.727, 0.594, and 0.566. When used to predict LNM, the AUC of CEA, CA199 and CA125 were 0.696, 0.531, and 0.588. Logistic regression analysis demonstrated that preoperative serum CEA were significantly associated with positive LNM. On combining imaging examination with CEA, the sensitivity and specificity were 85.3 and $79.4 \%$, respectively, with the AUC equal to 0.853 . The combination of CEA and imaging examination preformed the highest levels of AUC and sensitivity for diagnosing LNM, which is significantly higher than using either of them alone. Although patients with abnormal CEA have a poor prognosis, two models of multivariate analysis showed that CEA was not the independent prognosis factor for survival.

Conclusions: CEA can be used to diagnose gastric cancer and determine whether it has LNM. Moreover, combined with CEA could improve the diagnostic sensitivity of imaging examination for lymph node involvement.

Keywords: Carcinoembryonic antigen, Gastric cancer, Lymph node metastasis, Prognosis, Imaging examination

\footnotetext{
* Correspondence: jiaozy@lzu.edu.cn

${ }^{\dagger}$ Keshen Wang and Xiangyan Jiang contributed equally to this work.

'Department of General Surgery, Lanzhou University Second hospital, Cheng-Guan District, Lanzhou, Gansu 730030, China

${ }^{2}$ Cui-ying Experimental Center, Lanzhou University Second hospital,

Cheng-Guan District, Lanzhou 730030, Gansu, China
}

C C The Author(s). 2020 Open Access This article is licensed under a Creative Commons Attribution 4.0 International License, which permits use, sharing, adaptation, distribution and reproduction in any medium or format, as long as you give appropriate credit to the original author(s) and the source, provide a link to the Creative Commons licence, and indicate if changes were made. The images or other third party material in this article are included in the article's Creative Commons licence, unless indicated otherwise in a credit line to the material. If material is not included in the article's Creative Commons licence and your intended use is not permitted by statutory regulation or exceeds the permitted use, you will need to obtain permission directly from the copyright holder. To view a copy of this licence, visit http://creativecommons.org/licenses/by/4.0/ The Creative Commons Public Domain Dedication waiver (http://creativecommons.org/publicdomain/zero/1.0/) applies to the data made available in this article, unless otherwise stated in a credit line to the data. 


\section{Background}

Gastric cancer (GC) is the fourth most common malignancy worldwide and the second leading cause of cancer-associated mortality [1]. Because most of patients are in an advanced stage at the time of diagnosis, the mortality rates for GC have continued to increase in the past decade, especially in China [2]. The reason for this may be tumour invasion or, lymph node metastasis (LNM), in addition to other factors such as differentiation, genetic mutation and patient behaviour, and postoperative recurrence and metastasis [3]. Therefore, it is important to keep looking for new prognostic factors to help in the selection of reasonable treatment strategies.

Previous studies have found that several factors have been associated with the prognosis of patients with GC, including tumour size, differentiation, lymph node metastasis (LNM) and selection of treatment. Among these factors, lymph node status may be the most reliable prognostic factor accessible [4-7]. Regional LNM is assessed via CT, MRI, or pathologic analysis, and a positive result for LNM is defined as the presence of any lymph nodes with disease. In addition, LNM is broadly recognized as an indicator of tumour progression and prognosis in GC patients following curative gastrectomy [4]. At present, D2 lymphadenectomy is the main surgery for advanced gastric cancer (AGC) as well as the majority of submucosal cancers [8]. However, it is unreasonable blindly carry out D2 lymphadenectomy in patients without LNM (N0) or with only N1 stage metastasis (N1) [9]. Therefore, accurately predicting the lymph node statue is important for selecting the optimal surgical methods preoperatively. In recent years, preoperative detection of LNM has depended on imaging studies, such as contrast-enhanced computed tomography (CECT), upper endoscopy, and magnetic resonance imaging (MRI). Nevertheless, it is very challenging for these conventional modalities to accurately detect LNM because of their low sensitivities and specificities $[10,11]$. Therefore, additional methods are necessary to detect LNM before operation when imaging studies are unavailable or the results are not accurate.

Since carcinoembryonic antigen (CEA), one of the most common tumour markers, is known to reflect the clinical tumour burden in GC, it might be helpful in detecting positive lymph nodes [3, 12, 13]. However, there have been few reports on the prediction of LNM in LAGC using preoperative serum CEA. However, because early GC and LAGC are significantly different in terms of lymph node statue and survival, the predictive value of CEA for determining LNM may be low if the two stages are not separated. In addition, whether preoperative CEA levels can predict the survival of patients with $\mathrm{GC}$ is still controversial [11, 14-20].
The purpose of the present study was to investigate the predictive value of preoperative serum CEA for determining LNM, and to compare its sensitivity and specificity with those of imaging examination for detecting LNM, to explore the relationship between preoperative serum CEA and survival in patients with LAGC.

\section{Methods \\ Patients}

Between January 2013 and January 2018, 276 patients who were diagnosed with LAGC after surgical resection in our institution were enrolled in this retrospective study. The inclusion criteria were as follows: 1) pT24NxM0 resectable GC; 2) patients with histological confirmation of adenocarcinoma; 3) patients with a score of 0-2 on according to Zubrod-ECOG-WHO criteria; 4) patients with complete D2 or extend D2 lymphadenectomy; 5) patients with negative resection margins (R0); and 6) patients with complete medical records. Patients with any pretreatments, including chemotherapy and radiotherapy, were excluded. All patients were followed up via posting letters or telephone calls until death or the cut-off date (the last follow-up was 1 December 2018). All follow-up findings were collected and recorded in the database. Informed consent from the patients was waived because of the retrospective nature of this study. In addition, 172 patients with gastric polyps, chronic gastritis and gastric ulcers diagnosed by gastroscopy or pathological examination during the same period were also enrolled as the benign lesion control group. The study was approved by the Ethics Committee of the Second Hospital of Lanzhou University.

\section{Detection of tumor markers}

Serum CEA, CA199, and CA125 concentrations were recorded from routine clinical testing. Serum CEA, CA199, and CA125 were quantitatively measured using electro chemiluminescence immunoassay (ECLIA) kits (Roche Diagnostics Gmbh, Germany). The recommended upper cut-off values for CEA, CA199, and CA125 were $3.4 \mathrm{ng} / \mathrm{ml}, 27 \mathrm{U} / \mathrm{ml}$, and $35 \mathrm{U} / \mathrm{ml}$. Testing values over the cut-off values were regarded as positive.

\section{Clinicopathological characteristics}

The clinicopathological characteristics included age, sex, tumour location, tumour size, Lauren classification, degree of differentiation, nerve invasion, vessel invasion, tumour invasion, determination of LNM via CT or MRI, and determination of LNM via pathological were collected. Depth of tumour invasion was utilized to stage tumours according to the 7 th edition UICC guidelines [21]. 


\section{Statistical analysis}

Data are presented as the mean \pm standard derivation for normally distributed continuous data, as the median (interquartile range, Q25 - Q75) for abnormally distributed continuous data, and as actual values for categorical data. Comparisons between two groups were performed using Student's t test, a Wilcoxon test, or a chi-square test. The value of the three tumour markers for the diagnosis of GC and evaluation of LNM were calculated using the receiver operating characteristic (ROC) curve, which was used to distinguish the optimal cut-off value, accuracy, positive predictive value, and negative predictive value of each marker by calculating the max Youden index (sensitivity + specificity -1$)$. The area under the curve (AUC) was compared using the McNemar test. The potential risk factors for predicting LNM were determined by logistic regression analysis. Kaplan-Meier analysis with a log-rank test was used to calculate the overall cumulative probability, and the independent prognostic factors were identified by multivariate COX regression analysis. The primary outcome was OS, which was defined as the interval from gastrectomy to death of all causes. A $P$ value less than 0.05 was considered statistically significant. All statistical analyses were conducted using Statistical Product for Social Sciences (SPSS) software (version 23.0; SPSS Inc., Chicago, USA).

\section{Results}

A total of 448 patients were included in our study, of which 276 were diagnosed with GC and 172 were diagnosed with benign gastric diseases. As shown in Table 1, the average age in the gastric cancer group was $57.28 \pm$ 9.9 years, and this group including 203 (73.55\%) males and 73 (26.45) females. The average age of patients in the benign gastric disease group was $55.74 \pm 13.35$ years, and this group including 97 (56.4\%) males and 75 (43.6\%) females. The GC group had more males, higher CEA $(2.74 \mathrm{ng} / \mathrm{ml}, \quad 1.685-5.62)$, CA199 $(10.19 \mathrm{U} / \mathrm{ml}$, 6.12-19.49), and CA125 (12.025 U/ml, 8.662-19.23) levels than the benign gastric disease group. To estimate the ability of the three tumour markers to distinguish $\mathrm{GC}$ from benign gastric disease, ROC curves were generated, and the results showed that the area under the curve (AUC) values for CEA, CA199, and CA 125 were $0.727(0.681-0.773), 0.594(0.54-0.648)$, and $0.566(0.513-$
0.618), respectively, with optimal cut-off values of 1.95 $\mathrm{ng} / \mathrm{ml}, 17.12 \mathrm{U} / \mathrm{ml}, 9.675 \mathrm{U} / \mathrm{ml}$ (Fig. 1a, Table 2). When using the common cut-off value of $3.4 \mathrm{ng} / \mathrm{ml}$ for CEA, $27 \mathrm{U} / \mathrm{ml}$ for CA199, and $35 \mathrm{U} / \mathrm{ml}$ for CA125, the AUC values were $0.614,0.484$, and 0.4 , respectively (Table 2 ). This finding indicated that serum CEA, CA199, and CA125 values have the ability to diagnose GC.

\section{The value of preoperative clinical characteristics and tumor markers in predicting LNM}

Since the three tumour markers showed definite value in the diagnosis of GC, we further investigated whether they had the same value in the diagnosis of lymph node involvement. The ROC curves showed that the AUC values of CEA, CA199, and CA125 were 0.696 (0.634$0.759), 0.531(0.461-0.601)$, and $0.588(0.517-0.66)$, respectively (Fig. 1b). As shown in Table 3, LNM based on pathology assessment was associated with higher CEA $(P<0.001)$, higher CA199 $(P=0.001)$, and LNM based on imaging examination (Table 3). Multivariate logistic regression analysis was applied to determine the independent risk factors for LNM, and the results demonstrated that preoperative CEA (OR,4.86; 95\%CI 2.3310.139; $P<0.001)$ and LNM base on CT or MRI (OR, 47.81, 95\%CI 16.34-139.9; $P<0.001)$ independently affected LNM (Table 3).

\section{The performance of serum CEA compared with that of imaging examination in determining LNM}

Based on the cut-off value of $3.4 \mathrm{ng} / \mathrm{ml}$ used commonly in the clinic, the AUC of CEA for predicting LNM in the whole cohort was 0.677. Meanwhile, the AUC of imaging examination alone, including CT and MRI, was 0.823. The combination of CEA and imaging examination predicted the highest value of positive lymph nodes, which is significantly higher than the value using either of strategy alone (Table 4). Although imaging examination combined with preoperative serum CEA level showed the highest sensitivity (0.853) and accuracy (0.833), the imaging examination showed the highest specificity $(0.957)$ and positive predictive value $(0.97)$ for prediction the LNM.

Table 1 Comparison of clinical features and tumor markers between two groups

\begin{tabular}{llll}
\hline Variables & Gastric cancer group $(n=276)$ & Benign lesion group $(n=172)$ & $P$ value \\
\hline Age (years) & $57.28 \pm 9.9$ & $55.74 \pm 13.35$ & 0.166 \\
Sex $($ Male, \%) & $203(73.55 \%)$ & $97(56.4 \%)$ & 0.001 \\
CEA $(\mathrm{ng} / \mathrm{ml})$ & $2.74(1.685-5.62)$ & $1.525(1.09-2.385)$ & $<0.001$ \\
CA199 $(\mathrm{U} / \mathrm{ml})$ & $10.19(6.12-19.49)$ & $8.95(5.895-13.29)$ & 0.019 \\
CA125 $(\mathrm{U} / \mathrm{ml})$ & $12.025(8.662-19.23)$ & $9.99(7.447-14.8)$ & 0.001 \\
\hline
\end{tabular}



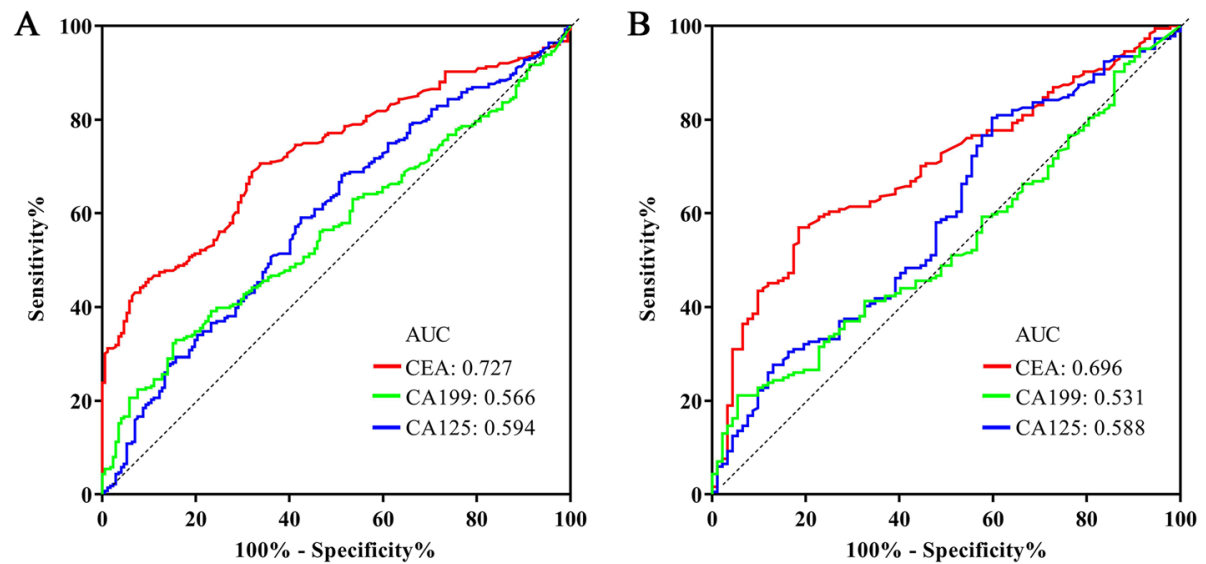

Fig. 1 The results of ROC curve analysis for the power of CA199, CA125, and CEA in diagnosing GC and LNM. a ROC curve of CA199, CA125, and CEA in diagnosing GC form gastric benign lesion. b ROC curve of CA199, CA125, and CEA in diagnosing LNM in patients with LAGC

\section{Predictive value of CEA for LNM in subgroups}

Although CEA performed well in predicting LNM in the entire population, we noted that 163 (59\%) patients did not have an elevated CEA $(\leq 3.4 \mathrm{ng} / \mathrm{ml})$. Therefore, we divided patients into two groups, the CEA positive group $(>3.4 \mathrm{ng} / \mathrm{ml})$ and CEA negative group $(\leq 3.4 \mathrm{ng} / \mathrm{ml})$, and investigated the diagnostic performance of CEA for LNM respectively in these two groups. As shown in Table 5, the AUC of CEA was 0.623(0.467-0.78) in the CEA positive group and merely $0.521(0.432-0.61)$ in the CEA negative group. Meanwhile, the accuracy, sensitivity and positive predictive value of CEA in prediction LNM were higher in the CEA positive group than these in the CEA negative group (accuracy, 0.611 vs 0.509 ; sensitivity, 0.588 vs 0.09 ; positive predictive value: 0.934 vs 0.889 ), indicating that CEA has limited predictive value for LNM in those patients with CEA negativity. We also performed further analyses that in the CEA-positive group and found that, the optimal cut-off value of CEA was $7.13 \mathrm{ng} / \mathrm{ml}$, the corresponding sensitivity was 0.588 , and the specificity was 0.75 .

\section{Survival analysis}

The median OS of CEA-positive group $(n=113)$ and CEA-negative group $(n=163)$ were 40 months and 21 months $(P=0.014)$, respectively (Fig. 2a). Univariate analysis showed that Lauren classification $(P=0.032)$, nerve invasion $(P=0.017)$, vessel invasion $(P=0.006)$, differentiation degree $(P=0.009)$, tumor size $(P<0.001)$, CEA $\quad(P=0.016), \quad$ CA199 $\quad(P<0.001), \quad$ pT $\quad$ stage $(P<0.001)$, and LNM based on pathology $(P<0.001)$ were significantly association with prognosis (Table 6). Further multivariate analysis demonstrated that preoperative CA199 level (HR, 1.608; 95\%CI 1.051-2.46; $P=0.028$ ), LNM based on pathology (HR:3.661, 95\%CI: $2.079-6.446, P=0.001)$ and $\mathrm{pT}$ stage $(P=0.046)$ were independent prognostic factors for the patients with LAGC (Table 6). The results of the X-tile plots demonstrated that the optimal cut-off point for CEA in OS prediction was $7.2 \mathrm{ng} / \mathrm{ml}$, with a $\times 2$ value of 16.7 , a $P$ value of 0.001 , and a relative risk ratio of 1:1.59 (Fig. 2b, c). Then multivariate analysis was performed again with the level of CEA stratified by $7.2 \mathrm{ng} / \mathrm{ml}$ instead of $3.4 \mathrm{ng} / \mathrm{ml}$, and the results also showed that serum CEA was not an independent factor for OS ((Table 6).

\section{Discussion}

Because GC is the second leading cause of cancerrelated death around the world, its diagnosis and treatment are constantly attracting much attention [1]. The

Table 2 Diagnostic performances of three serum tumor markers for differentiating GC from benign gastric disease

\begin{tabular}{llllllll}
\hline Group & Cut-off value & Sensitivity & Specificity & Positive predictive value & Negative predictive value & Accuracy & AUC (95\%Cl) \\
\hline CEA & $1.95 \mathrm{ng} / \mathrm{ml}$ & 0.707 & 0.663 & 0.771 & 0.585 & 0.69 & $0.727(0.681-0.773)$ \\
& $3.4 \mathrm{ng} / \mathrm{ml}$ & 0.409 & 0.942 & 0.919 & 0.499 & 0.614 & $0.676(0.627-0.725)$ \\
CA199 & $17.12 \mathrm{U} / \mathrm{ml}$ & 0.843 & 0.33 & 0.771 & 0.439 & 0.527 & $0.566(0.513-0.619)$ \\
& $27 \mathrm{U} / \mathrm{ml}$ & 0.199 & 0.942 & 0.846 & 0.423 & 0.484 & $0.571(0.517-0.624)$ \\
CA125 & $9.675 \mathrm{U} / \mathrm{ml}$ & 0.488 & 0.681 & 0.681 & 0.488 & 0.607 & $0.594(0.540-0.648)$ \\
& $35 \mathrm{U} / \mathrm{ml}$ & 0.843 & 0.33 & 0.667 & 0.386 & 0.4 & $0.505(0.45-0.56)$ \\
\hline
\end{tabular}


Table 3 Logistic regression analysis of preoperative factors predicting LNM based on pathology in patients with LAGC

\begin{tabular}{|c|c|c|c|c|c|c|c|}
\hline \multirow[t]{2}{*}{ Variables } & & \multicolumn{3}{|c|}{ Univariate analysis } & \multicolumn{3}{|c|}{ Multivariate analysis } \\
\hline & & LNM -, n (\%) & LNM +, n (\%) & $p$ value & $\overline{O R}$ & $95 \% \mathrm{Cl}$ & $p$ value \\
\hline \multirow[t]{2}{*}{ Gender } & Male & $66(71.7)$ & $137(74.5)$ & 0.665 & & & \\
\hline & female & $26(28.3)$ & $47(25.5)$ & & & & \\
\hline \multirow[t]{2}{*}{ Age (years) } & $\leq 60$ & $50(54.3)$ & 113(61.4) & 0.299 & & & \\
\hline & $>60$ & $42(45.7)$ & $71(38.6)$ & & & & \\
\hline \multirow[t]{3}{*}{ Tumor location } & Upper & $16(17.4)$ & $46(25)$ & 0.146 & & & \\
\hline & Middle & $50(54.3)$ & $78(42.4)$ & & & & \\
\hline & lower & $26(28.3)$ & $60(32.6)$ & & & & \\
\hline \multirow[t]{2}{*}{ CA199 (U/ml) } & $\leq 37$ & 87(94.6) & 147(79.9) & 0.001 & 1 & & \\
\hline & $>37$ & $5(5.4)$ & $37(20.1)$ & & 2.265 & $0.672-7.632$ & 0.187 \\
\hline \multirow[t]{2}{*}{ CA125 (U/ml) } & $\leq 35$ & $90(97.8)$ & 172(93.5) & 0.152 & & & \\
\hline & $>35$ & $2(2.2)$ & $12(6.5)$ & & & & \\
\hline \multirow[t]{2}{*}{ CEA (ng/ml) } & $\leq 3.4$ & 76(82.6) & $87(47.3)$ & $<0.001$ & 1 & & $<0.001$ \\
\hline & $>3.4$ & 16(17.4) & $97(52.7)$ & & 4.86 & $2.33-10.139$ & \\
\hline \multirow[t]{2}{*}{ LNM base on CT or MRI } & No & $88(95.7)$ & $57(31)$ & $<0.001$ & 1 & & $<0.001$ \\
\hline & Yes & $4(4.3)$ & $127(69)$ & & 47.81 & $16.34-139.9$ & \\
\hline
\end{tabular}

majority of Chinese patients with GC are at an advanced stage at the time of diagnosis, and they often have perigastric LNM involvement, which is widely considered a significant prognostic factor and a basis for treatment decisions in GC patients [4-8]. However, appropriate preoperative assessment of lymph node status is still difficult and conventional methods, such as CECT and MRI, are limited in detecting LNM due to their low sensitivity in lymph nodes less than $0.5 \mathrm{~cm}$ and their low sensitivity in distinguishing cancer from inflammatory hyperplasia. Thus, it is important to determine a feasible method to use in combination with conventional approaches to accurately detect preoperative LNM and develop individualized treatment protocols.

Currently, CEA, a carcino-embryonic antigen located on chromosome 19, is a commonly used tumour marker in the diagnosis of malignant tumours of the digestive tract, and high levels of CEA are closely associated with tumor burden [14, 17, 22]. Therefore, we hypothesized that CEA would be helpful for the determination of LNM in GC patients. Before we tested this, we first tested the ability of CEA to diagnose GC, because if an elevated CEA was not successful in differentiating from
GC benign processes, its value for diagnosing LNM in GC would likely be limited. In this study, the median value of CEA in the GC group was 2.74 (1.685-5.62) ng/ $\mathrm{ml}$, which was higher than the $1.525(1.09-2.385) \mathrm{ng} / \mathrm{ml}$ in the benign control, and the difference was statistically significant. When we used the clinically recommended $3.4 \mathrm{ng} / \mathrm{ml}$ as the cut-off value, the sensitivity was 0.409 , the specificity was 0.942 , and the AUC was 0.676 , which are consistent with previous research [23, 24]. Therefore, we believe that the level of CEA can help diagnose GC.

In regard to determining LNM, our data show that the percentage of samples with elevated CEA, CA199, and CA125 in the LNM-positive group was significantly higher than that in the LNM-negative group. This suggests that elevated levels of CEA, CA199, and CA125 may be associated with lymph node involvement. In the multivariate regression analysis of these three tumour markers, only CEA was an independent factor in the determination of LNM. However, there have been few studies regarding the value of preoperative CEA in evaluating LNM in GC. M Ikeguchi et al. [25] reported that elevated levels of CEA before surgery were a good indicator of LNM in patients with GC. Li et al. [26]

Table 4 Diagnostic efficiency of conventional methods, CEA used alone and their combined use for distinguishing lymph node metastasis from gastric cancer patients

\begin{tabular}{|c|c|c|c|c|c|c|c|}
\hline Methods & Sensitivity & Specificity & Positive predictive value & Negative predictive value & Accuracy & AUC $(95 \% \mathrm{Cl})$ & $P$ value \\
\hline CEA $>3.4 \mathrm{ng} / \mathrm{ml}$ & 0.527 & 0.826 & 0.858 & 0.466 & 0.627 & $0.677(0.612-0.742)$ & $<0.001^{\mathrm{a}}$ \\
\hline Imaging examination & 0.690 & 0.957 & 0.97 & 0.607 & 0.779 & $0.823(0.774-0.873)$ & $<0.001^{\mathrm{b}}$ \\
\hline Imaging examination + CEA & 0.853 & 0.794 & 0.892 & 0.73 & 0.833 & $0.88(0.843-0.918)$ & \\
\hline
\end{tabular}

a CEA vs CEA + Imaging examination

${ }^{b}$ Imaging examination vs Imaging examination + CEA 
Table 5 Subgroup analysis of CEA predictive value for lymph node metastasis

\begin{tabular}{llllllll}
\hline Group & Optimal cut-off value & Sensitivity & Specificity & Positive predictive value & Negative predictive value & Accuracy & AUC (95\%Cl) \\
\hline CEA $>3.4 \mathrm{ng} / \mathrm{ml}$ & $7.13 \mathrm{ng} / \mathrm{ml}$ & 0.588 & 0.75 & 0.934 & 0.231 & 0.611 & $0.623(0.467-0.78)$ \\
CEA $\leq 3.4 \mathrm{ng} / \mathrm{ml}$ & $3.21 \mathrm{ng} / \mathrm{ml}$ & 0.09 & 0.987 & 0.889 & 0.487 & 0.509 & $0.521(0.432-0.61)$
\end{tabular}

reported that preoperative serum CEA was significantly associated with positive lymph node count, and its correlation coefficient was higher than CA199 and CA724. In this study, based on the common cut-off value of $3.4 \mathrm{ng} / \mathrm{ml}$, the sensitivity and specificity of CEA for determining LNM were 0.527 and 0.826 , respectively, which were consistent with those found in a previous study [27]. To investigate the diagnostic performance of CEA, CEA levels and imaging results were first compared in our study. The results demonstrated that although the ability to determine LNM using CEA alone was significantly lower than that using imaging examination, a combination of these two methods showed the highest sensitivity, accuracy, and AUC. This indicated that CEA can assist CT or MRI to better detect lymph node status preoperatively, and added weight to show the importance of preoperative serum CEA for determining LNM. One meta-analysis reported that due to the different standard values applied by institutions, types of antibodies used, tumour stages, sensitivities and specificities, the rate of positive serum CEA results ranged from 2.3 to $60.82 \%$ [14]. It seems that the presence of CEA in the serum is dependent on increased production of CEA by cancer cells. Our data showed that $41 \%$ patients had an abnormal CEA value, so we performed a subgroup analysis to assess the performance of CEA in determining LNM in the CEA-positive group and the normal group. We found that in the CEA-positive group, the predictive power of CEA for determining LNM was strong, with the AUC was 0.623. When the cut-off value was set at $7.12 \mathrm{ng} / \mathrm{ml}$ according to the max value of the Youden index, the sensitivity was 0.588 , specificity was 0.75 , and accuracy was equaled to 0.611. Nevertheless, in the CEA-normal group, the AUC was merely 0.521 , which indicated that when the CEA in the serum of GC patients does not increase, its ability to determine LNM is also limited. This finding needs to be validated in subsequent large sample studies.

In previous studies, it has been reported that CEA in serum is an independent prognostic factor, while CEA in tumour tissues is not [15]. Uda et al. [16] performed a retrospective study with a median follow-up of 39.6 months in 251 T2-4 GC patients. The 5-year survival rate was significantly lower in the preoperative CEA-
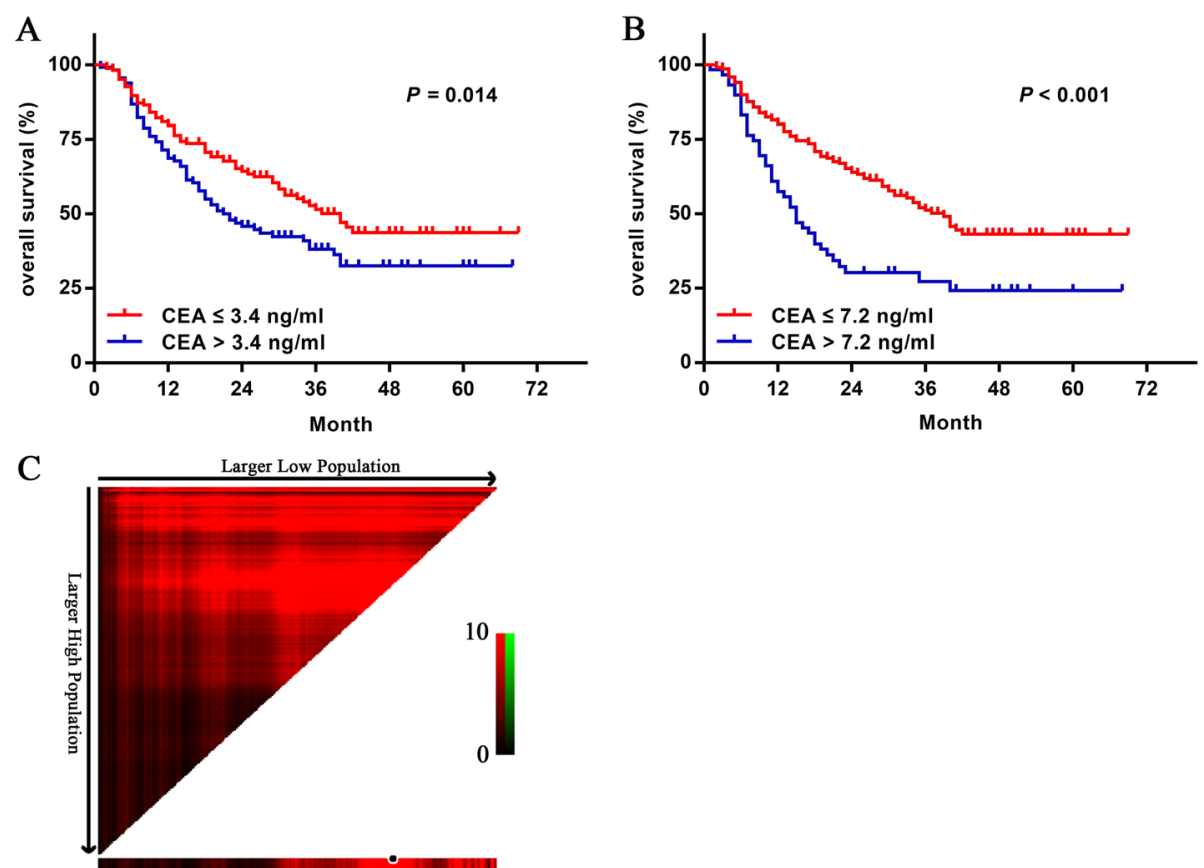

Fig. 2 Survival analysis in 267 patients with LAGC and patients divided by X-tile plot. a Kaplan-Meier curves for OS when the cutoff value of CEA is $3.4 \mathrm{ng} / \mathrm{ml}$. $\mathbf{b}$ Kaplan-Meier curves for OS when the cutoff value of CEA is $7.2 \mathrm{ng} / \mathrm{ml}$. c Division of patients by the cut-off points calculated by X-tile plot 
Table 6 univariate and multivariate analysis for the entire patients with locally advanced gastric cancer

\begin{tabular}{|c|c|c|c|c|c|c|c|c|c|}
\hline \multirow[b]{2}{*}{ Variables } & \multicolumn{3}{|c|}{ Univariate analysis } & \multicolumn{3}{|c|}{${ }^{a}$ Multivariate analysis } & \multicolumn{3}{|c|}{${ }^{\mathrm{b}}$ Multivariate analysis } \\
\hline & $H R$ & $95 \% \mathrm{Cl}$ & p & $H R$ & $95 \% \mathrm{Cl}$ & $p$ & $H R$ & $95 \% \mathrm{Cl}$ & $p$ \\
\hline Gender (female) & 0.857 & $0.584-1.258$ & 0.431 & & & & & & \\
\hline Age (> 60 years) & 1.067 & $0.764-1.489$ & 0.705 & & & & & & \\
\hline Lauren classification & & & 0.032 & & & 0.245 & & & 0.132 \\
\hline Intestinal & 1 & & & 1 & & & 1 & & \\
\hline Diffused & 1.658 & $1.128-2.437$ & & 1.363 & $0.853-2.179$ & & 1.431 & $0.886-2.309$ & \\
\hline Mixed & 1.45 & $0.94-2.237$ & & 0.961 & $0.581-1.589$ & & 0.929 & $0.568-1.519$ & \\
\hline Nerve invasion (yes) & 1.675 & $1.094-2.51$ & 0.017 & 1.098 & $0.633-1.903$ & 0.74 & 1.068 & $0.612-1.863$ & 0.816 \\
\hline Vessel invasion (yes) & 2.07 & $1.228-3.487$ & 0.006 & 1.008 & $0.505-2.01$ & 0.983 & 1.102 & $0.555-2.191$ & 0.781 \\
\hline Tumor location & & & 0.916 & & & & & & \\
\hline Upper & 1 & & & & & & & & \\
\hline Middle & 0.979 & 0.634 & & & & & & & \\
\hline Lower & 1.062 & $0.67-1.682$ & & & & & & & \\
\hline Differentiation degree (well) & 0.638 & $0.455-0.895$ & 0.009 & 0.747 & $0.487-1.144$ & 0.18 & 0.74 & $0.481-1.138$ & 0.17 \\
\hline Tumor size (> $4 \mathrm{~cm})$ & 1.891 & $1.353-2.644$ & $<0.001$ & 1.371 & $0.961-1.956$ & 0.082 & 1.293 & $0.899-1.859$ & 0.166 \\
\hline CEA (> $3.4 \mathrm{ng} / \mathrm{ml})$ & 1.507 & $1.081-2.102$ & 0.016 & 1.097 & $0.75-1.604$ & 0.632 & & & \\
\hline CEA (> $7.2 \mathrm{ng} / \mathrm{ml})$ & & & & & & & 1.4 & $0.919-2.132$ & 0.117 \\
\hline CA199 (> 37 U/ml) & 2.089 & $1.4-3.116$ & $<0.001$ & 1.608 & $1.051-2.46$ & 0.028 & 1.492 & $0.978-2.274$ & 0.063 \\
\hline CA125 (> $35 \mathrm{U} / \mathrm{ml})$ & 1.776 & $0.933-3.382$ & 0.08 & 1.223 & $0.632-2.369$ & 0.55 & 1.038 & $0.519-2.078$ & 0.915 \\
\hline LNM based on pathology (yes) & 4.758 & $2.896-7.816$ & $<0.001$ & 3.661 & $2.079-6.446$ & 0.001 & 3.38 & $1.937-5.898$ & 0.001 \\
\hline pT stage & & & $<0.001$ & & & 0.04 & & & 0.074 \\
\hline $\mathrm{T} 2$ & 1 & & & 1 & & & 1 & & \\
\hline T3 & 2.627 & $1.008-6.843$ & & 1.163 & $0.414-3.266$ & & 1.123 & $0.4-3.154$ & \\
\hline T4 & 5.682 & $2.314-13.951$ & & 2.021 & $0.727-5.617$ & & 3.38 & $1.937-5.898$ & \\
\hline
\end{tabular}

${ }^{\text {aUsing }} 3.4 \mathrm{ng} / \mathrm{ml}$ to stratify CEA

bUsing $7.2 \mathrm{ng} / \mathrm{ml}$ to stratify CEA

positive group than in the CEA-negative group, and this difference was further confirmed in the multivariate analysis. In addition, the results of a meta-analysis also showed that preoperative serum CEA was an independent prognostic factor for GC [14]. However, Ucar et al. [17] reported that preoperative serum CA724, rather than CEA, was an independent factor affecting the prognosis of GC. The results of Duraker [18] also showed that preoperative serum CEA and CA199 were not independent prognostic factors for GC. In the current study, although the univariate analysis revealed that a CEA value greater than $3.4 \mathrm{ng} / \mathrm{ml}$ was associated with unfavourable survival, the findings of the multivariate analysis showed that a CEA cut-off value of $3.4 \mathrm{ng} / \mathrm{ml}$ did not allow CEA to function as an independent poor prognostic factor. Next, we also used X-plot to determine the best cut-off value of CEA for predicting prognosis, and found it to be $7.2 \mathrm{ng} / \mathrm{ml}$. This cutoff value was consistent with the the previous literature reported [15]. We performed multivariate regression analysis with CEA cut-off value of $7.2 \mathrm{ng} /$ $\mathrm{ml}$ instead of $3.4 \mathrm{ng} / \mathrm{ml}$ and found that CEA was still not an independent prognostic factor.

Of note, our research does have some limitations. First, this study with a retrospective nature and was performed at a single centre, which inevitably led to potential biases and a relatively small sample size. Second, the positive rate of tumour markers in GC was relatively low, which may have resulted in bias during the analysis. Third, because the patients were consecutively enrolled, some patients had a follow-up period of less than 5 years and no associated outcomes, which may had an impact on the results of the survival analysis.

\section{Conclusions}

For patients with LAGC, preoperative CEA is a strong factor for determining LNM and may be useful for improving the sensitivity of conventional methods in determining LNM when the two approaches are combined. Serum CEA can be used for the diagnosis of GC and can assist in predicting prognosis, but it is not an independent prognostic factor. 


\section{Abbreviations}

GC: Gastric cancer; LAGC: Locally advanced gastric cancer;

CEA: Carcinoembryonic antigen; CA125: Carbohydrate antigen 125; CA199: Carbohydrate antigen 199; TNM: Tumor, Lymph nodes, Metastasis: CECT: Contrast-enhanced computed tomography; MRI: Magnetic resonance imaging; LNM: Lymph node metastasis; ROC: Receiver operating characteristics; AUC: Area under the ROC curve

\section{Acknowledgements}

Not Applicable.

\section{Authors' contributions}

KSW and XYJ conceived and written the study; YXR, FL, JYX, XCC, and ZYY carried out the data collection; ZJM and JYX followed up patients; KSW and $X Y J$ conducted the data analysis; ZYY and ZYJ revised the paper; KSW and $X Y J$ are co-first authors. All authors have read and approved the final version of this manuscript.

\section{Funding}

This work was supported by the Fundamental Research Funds for the Central Universities (Izujbky-2019-cd06).

\section{Availability of data and materials}

The datasets generated or analyzed during the current study are available from the corresponding author on reasonable request.

\section{Ethics approval and consent to participate}

This study was conducted in accordance with the declaration of Helsinki. Informed consent from the patients was waived because of the retrospective nature of this study, and this study was approved by Institutional Ethnic Committee of the Lanzhou University second hospital (2018A-090).

\section{Consent for publication}

Not Applicable.

\section{Competing interests}

All authors declare no conflicts of interest.

Received: 23 December 2018 Accepted: 31 March 2020 Published online: 10 April 2020

\section{References}

1. Sitarz R, Skierucha M, Mielko J, Offerhaus GJA, Maciejewski R, Polkowski WP. Gastric cancer: epidemiology, prevention, classification, and treatment. Cancer Manag Res. 2018;10:239-48.

2. Patru CL, Surlin V, Georgescu I, Patru E. Current issues in gastric cancer epidemiology. Rev Med Chir Soc Med Nat lasi. 2013;117(1):199-204.

3. Tsai YC, Hsiao WH, Lin SH, Yang HB, Cheng HC, Chang WL, Lu CC, Sheu BS. Genomic single nucleotide polymorphisms in the offspring of gastric cancer patients predispose to spasmolytic polypeptide-expressing metaplasia after H. pylori, infection. J Biomed Sci. 2015;22:16.

4. Kutlu OC, Watchell M, Dissanaike S. Metastatic lymph node ratio successfully predicts prognosis in Western gastric cancer patients. Surg Oncol. 2015; 24(2):84-8.

5. Siewert JR, Böttcher K, Stein HJ, Roder JD. Relevant prognostic factors in gastric cancer: ten-year results of the German gastric Cancer study. Ann Surg. 1998;228(4):449-61.

6. Fukuda N, Sugiyama Y, Midorikawa A, Mushiake H. Prognostic significance of the metastatic lymph node ratio in gastric cancer patients. World J Surg. 2009;33(11):2378-82.

7. Celen O, Yildirim E, Berberoglu U. Prognostic impact of positive lymph node ratio in gastric carcinoma. J Surg Oncol. 2007;96(2):95-101.

8. Roviello F, Rossi S, Marrelli D, Pedrazzani C, Corso G, Vindigni C, Morgagni P, Saragoni L, de Manzoni G, Tomezzoli A. Number of lymph node metastases and its prognostic significance in early gastric cancer: a multicenter Italian study. J Surg Oncol. 2006;94(4):275-80.

9. Kitagawa Y, Takeuchi H, Takagi Y, Natsugoe S, Terashima M, Murakami N, Fujimura T, Tsujimoto H, Hayashi H, Yoshimizu N, et al. Sentinel node mapping for gastric cancer: a prospective multicenter trial in Japan. J Clin Oncol. 2013;31(29):3704-10.
10. Kudou M, Kosuga T. Kubota T1, Okamoto K, Komatsu S, Shoda K, Konishi H, Shiozaki a, Fujiwara H, Arita T, et al. value of preoperative PET-CT in the prediction of pathological stage of gastric Cancer. Ann Surg Oncol. 2018; 25(6):1633-9.

11. Kwee RM, Kwee TC. Imaging in assessing lymph node status in gastric cancer. Gastric Cancer. 2009;12(1):6-22.

12. Mihmanli M, Dilege E, Demir U, Coskun H, Eroglu T, Uysalol MD. The use of tumor markers as predictors of prognosis in gastric cancer. Hepatogastroenterology. 2004;51(59):1544-7.

13. Ni XG, Bai XF, Mao YL, Shao YF, Wu JX, Shan Y, Wang CF, Wang J, Tian YT, Liu $Q$, et al. The clinical value of serum CEA, CA19-9, and CA242 in the diagnosis and prognosis of pancreatic cancer. Eur J Surg Oncol. 2005;31(2):164-9.

14. Deng K, Yang L, Hu B, Wu H, Zhu H, Tang C. The prognostic significance of pretreatment serum CEA levels in gastric Cancer: a meta-analysis including 14651 patients. PLoS One. 2015;10(4):e0124151.

15. Park SH, Ku KB, Chung HY, Yu W. Prognostic significance of serum and tissue Carcinoembryonic antigen in patients with gastric adenocarcinomas. Cancer Res Treat. 2008;40(1):16-21.

16. Uda H, Kanda M, Tanaka C, Kobayashi D, Inaoka K, Tanaka Y, Hayashi M, Iwata N, Yamada S, Fujii T, et al. Perioperative serum Carcinoembryonic antigen levels predict recurrence and survival of patients with pathological T2-4 gastric Cancer treated with curative Gastrectomy. Dig Surg. 2018;35(1):55-63.

17. Ucar E, Semerci E, Ustun H, Yetim T, Huzmeli C, Gullu M. Prognostic value of preoperative CEA, CA 19-9, CA 72-4, and AFP levels in gastric cancer. Adv Ther. 2008;25(10):1075-84.

18. Duraker N, Celik AN. The prognostic significance of preoperative serum CA 19-9 in patients with resectable gastric carcinoma: comparison with CEA. J Surg Oncol. 2001;76(4):266-71.

19. Chen S, Chen YB, Li YF, Feng XY, Zhou ZW, Yuan XH, Qian CN. Normal carcinoembryonic antigen indicates benefit from perioperative chemotherapy to gastric carcinoma patients. World J Gastroenterol. 2012; 18(29):3910-6.

20. Xiao J, Ye ZS, Wei SH, Zeng Y, Lin ZM, Wang Y, Teng WH, Chen LC. Prognostic significance of pretreatment serum carcinoembryonic antigen levels in gastric cancer with pathological lymph node-negative: a large sample single center retrospective study. World J Gastroenterol. 2017;23(48): 8562-9.

21. Edge SB, Byrd DR, Compton CC, et al. AJCC Cancer staging manual. 7th ed. New York: Springer; 2010.

22. Wu YC, Lv P, Han J, Yu JL, Zhu X, Hong LL, Zhu WY, Yu QM, Wang XB, et al. Enhanced serum methylated p16 DNAs is associated with the progression of gastric cancer. Int J Clin Exp Pathol. 2014;7(4):1553-62.

23. Wu D, Cao G, Huang Z, Jin K, Hu H, Yu J, Zeng Y. Decreased miR-503 expression in gastric cancer is inversely correlated with serum carcinoembryonic antigen and acts as a potential prognostic and diagnostic biomarker. Onco Targets Ther. 2016;10:129-35.

24. Wu Y, Jiang M, Qin Y, Lin F, Lai M. Single and combined use of neutrophillymphocyte ratio, platelet-lymphocyte ratio and carcinoembryonic antigen in diagnosing gastric cancer. Clin Chim Acta. 2018;481:20-4.

25. Ikeguchi M, Katano K, Saitou H, Tsujitani S, Maeta M, Kaibara N. Preoperative serum levels of CA72-4 in patients with gastric adenocarcinoma. Hepatogastroenterology. 1997:44(15):866-71.

26. Li F, Li S, Wei L, Liang X, Zhang H, Liu J. The correlation between preoperative serum tumor markers and lymph node metastasis in gastric cancer patients undergoing curative treatment. Biomarkers. 2013;18(7):632-7.

27. Wei X, Li YB, Li Y, Lin BC, Shen XM, Cui RL, Gu YJ, Gao M, Li YG, Zhang S. Prediction of lymph node metastases in gastric Cancer by serum APE expression. J Cancer. 2017:8(8):1492-7.

\section{Publisher's Note}

Springer Nature remains neutral with regard to jurisdictional claims in published maps and institutional affiliations. 